\title{
La tradición femenina en el siglo XVIII en Inglaterra
}

\section{Argentina RODRÍGUEZ \\ Universidad Nacional Autónoma de México}

\begin{abstract}
Este artículo explora las imágenes de la mujer en la Restauración y el siglo XVIII en Inglaterra con el propósito de establecer un continuum de la tradición literaria femenina en este siglo. Se examina la doble moral imperante, la situación social, política y económica de la mujer y el surgimiento de las primeras novelistas para arribar a una perspectiva feminista de la creación literaria de mujeres.
\end{abstract}

PALABRAS CLAVE: mujeres en la Inglaterra del siglo XVIII, perspectiva feminista del siglo XVIII en Inglaterra, tradición literaria de las mujeres del siglo XVIII en Inglaterra.

This essay explores he images of women in the Restoration period and Eighteenth century in England in order to establish a continuum of women's literary tradition in this century. The double standard, the social, political and economical situation of women are studied, as well as the rise of the first women novelists in the Eighteenth century. A feminist perspective is employed to examine the literary creation of women.

KEY WORDS: Women Eighteenth century England, Feminist perspective Eighteenth Century England, Women's literary tradition Eighteenth Century England.

For very little is known about women. The history of England is the history of the male line, not of the female. Of our fathers we know always some fact, some distinction. They were soldiers or they were sailors; they filled that office or they made that law. But of our mothers, our grandmothers, our great-grandmothers, what remains? Nothing but a tradition. One was beautiful; one was red-haired; one was kissed by a Queen. We know nothing of them except their names and the dates of their marriages and the number of children they bore.

Virginia Woolf, "Women and Fiction"

En el año 1811 se inicia, según la tradición crítica inglesa, el periodo de la novela escrita por mujeres. Es la publicación de Sense and Sensibility de Jane Austen el momento que marca la historia de la literatura como el hecho definitivo para la génesis de la novela femenina. Sin embargo, no podemos ignorar que más de ciento cincuenta años 
de producción femenina —obras de teatro, poesía, ensayos sobre conducta, educación, moral, filosofía, derechos de la mujer - rica en géneros y temáticas distintas van a allanar el camino de la Jane Austen novelista. De ahí mi interés en las escritoras poco conocidas, silenciadas, olvidadas por la crítica tradicional; como si Austen apareciera por generación espontánea, al margen de tantas otras escritoras que posibilitaron su "sentido" y su "sensibilidad". Es por ello que resulta indispensable para una correcta comprensión del fenómeno histórico que comienza con Jane Austen conocer la producción literaria de sus antecesoras, su visión del mundo y la significación de sus textos. Por lo mismo, nos atrevemos a afirmar que estudiar la novela inglesa implica remontarse hasta los finales del siglo XVII.

Es por esto que resulta relevante abordar temas como las imágenes de la mujer en el periodo de la Restauración y el siglo XVIII; el discurso de la Ilustración como un discurso que, a pesar de su pretensión universal, establece un doble discurso: el del hombre sobre el hombre y del hombre sobre la mujer, así como el double-standard o doble moral que juzga a la escritora con criterios distintos de los que se aplican al escritor. Un estudio sobre la mujer y la escritura nos remite necesariamente al surgimiento de la novela (the rise of the novel, como lo define el crítico inglés Ian Watt) y al papel de la mujer en el desarrollo de un nuevo género. Por lo tanto, nos interesa subrayar que la tradición literaria canónica se equivoca al borrar de un plumazo la voz de todo un conjunto de mujeres que posibilitaron a una Jane Austen y con ella el surgimiento de la novela, mujeres que podemos reconocer a partir de finales del siglo XVII hasta 1811. Entre ellas, podemos mencionar a Aphra Behn, Mary Astell, Mary Robinson, Eliza Haywood, Catherine Macaulay Graham, Priscilla Wakefield, Mary Hays, Hannah More, Susannah Centlivre y Mary Wollstonecraft.

La crítica feminista norteamericana Elaine Showalter plantea en su ensayo "Toward a Feminist Poetics" la necesidad de establecer una crítica feminista basada en la reconstrucción social, política y cultural de la experiencia de la mujer. Divide la crítica feminista en dos grandes grupos. El primero, woman as reader, aborda el estudio de la mujer como lectora de una literatura escrita por hombres; ésta se basa en imágenes y estereotipos femeninos, en la interpretación de la mujer por la crítica masculina. Esta aproximación, por lo tanto, omite a un gran número de escritoras en la historia de la literatura. Al segundo grupo lo nombra woman as writer, es decir, "woman as the producer of textual meaning, with the history, themes, genres, and structures of literature by women" (Showalter, “Toward": 128). Los temas de este análisis planteados por Showalter abarcan "the psychodynamics of female creativity, linguistics and the problem of a female language; the trajectory of the individual or collective female literary career; literary history; and, of course, studies of particular writers and works. No term exists in English for such a specialized discourse, and so I have adapted the French term la gynocritique: 'gynocritics"” (Showalter, “Toward”: 129).

A partir de la ginocrítica se pretende reconstruir un continuum de escritura femenina que abarca el periodo antes mencionado. La crítica masculina sólo se ha ocupado de algunas escritoras a las que juzga importantes, olvidando a otras que constituyen 
los eslabones de la cadena que une a una generación con otra. Éstas son las escritoras olvidadas, silenciadas, a quienes se les niega su lugar en un buen número de libros y antologías.

Si comenzamos a pensar en el periodo de la Restauración en Inglaterra vemos que las escritoras que se dedicaron a la literatura como profesión fueron objeto de burla y desprecio. Para estudiar a la escritora profesional de finales del siglo XVII y del XVIII, la que escribe para ganar dinero, se deben considerar ciertos aspectos de ese periodo en el que la literatura se convierte en un producto de consumo que depende del mercado. Al estudiar la tradición femenina de dichos siglos hallamos que en lo escrito por mujeres se subvierten las imágenes tradicionales, se crean relatos secretamente subversivos, superficies decorosas en las que irrumpe el deseo de igualdad, de libertad, de individualidad, relatos en los que cobra vida el lenguaje del silencio que se rehúsa a ser silenciado, que vuelve focal lo marginal. La característica de esta escritura es que se desarrolla dentro del discurso masculino dominante a través de actos de revisión, apropiación y subversión. A este respecto, Dale Spender, en Mothers of the Novel, señala: "By the time of the Restoration when more and more women were earning their living by the pen, the distinction between the prostitute and the woman writer was so blurred as to be almost non-existent and it is possible that the opprobium associated with both is more closely connected to the selling and the money making than it is to any particular commodity they were trying to sell" (Spender: 14).

En la segunda mitad del siglo XVIII muchas escritoras se interesan por cuestiones éticas, y entretejidos en sus narraciones hallamos sus planteamientos sobre moral, conducta y educación; sobre el individuo y su relación con la sociedad. Estas escritoras brindan opiniones y puntos de vista diferentes sobre la experiencia y la historia de la mujer. Pero hay que recordar que, desde una perspectiva actual, ser escritora en el siglo XVIII resulta en sí una contradicción. La mujer, quien carecía de derechos y, por tanto, de una existencia legal y moral como individuo, se expresa en el acto de escribir; su palabra se dirige a un público lector y a través de este acto logra afirmar su individualidad. Escribir equivale a ser, a crear y, por tanto, existir. Escribir significa construir una visión de mundo. Esta afirmación constituye una transgresión que la escritora disfraza, veladamente, y a través de las conductas que la sociedad considera apropiadas, cobra identidad. Al elegir la profesión de las letras debió inventar estrategias para defenderse de los ataques de una sociedad que la tachaba de transgresora o la condenaba al ridículo. Esto puede explicar por qué muchas se disculpan, en los prólogos y dedicatorias de sus obras, por la audacia, atrevimiento y presunción de escribir. Un ejemplo es Charlotte Lennox en el prefacio a su novela The Female Quixote: "The Dread which a Writer feels of the public Censure; the still greater Dread of Neglect; and the eager Wish for Support and Protection... are unknown to those who have never adventured into the World... 'Tis therefore, not unlikely, that the design of this Address may be mistaken, and the Effects of my Fear imputed to my Vanity" (Lennox, "Preface").

La falta de una educación formal es una realidad dolorosa que muchas mujeres comparten en el siglo XVIII. La educación, uno de los temas más debatidos, constituye 
uno de los medios para conservar la ideología imperante. Existen argumentos a favor de los derechos de la mujer a la educación y, desde la óptica de una política sexual, se plantea una cuestión muy importante que hasta el día de hoy continúa vigente: el tema de la diferencia. Varias escritoras critican los ensayos sobre conducta femenina y cuestionan la imagen de la feminidad creada por el hombre. En sus escritos rechazan la hipotética inferioridad e incapacidad de la mujer considerada "natural" a partir de la diferencia sexual. Mary Astell (1666-1731), escritora conservadora y religiosa que defiende la educación de la mujer, escribe en "A Serious Proposal to the Ladies, for the Advancement of their True and Greatest Interest" (1696):

Altho' it has been said by Men of more Wit and Wisdom, and perhaps of more malice than either, that Women are naturally incapable of acting Prudently, or that they are necessarily determined to folly, I must by no means grant it; that Hypothesis would render my endeavours impertinent, for then it would be in vain to advise the one, or endeavour the Reformation of the other. Besides, there are Examples in all Ages, which sufficiently confute the Ignorance and Malice of this Assertion.

The Incapacity, if there be any, is acquired, not natural; [...] Some disadvantages indeed they labour under, and what these are we shall see by and by and endeavour to surmount; but Women do not take up with mean things, since (if they are not wanting to themselves) they are capable of the best. Neither God nor Nature have excluded them from being Ornaments to their Families and useful in their Generation [...] The Cause therefore of the defects we labour under is, if not wholly, yet at least in the first place, to be ascribed to the mistakes of our Education, which like an Error in the first Concoction, spreads ill Influence through all our Lives (Perry: 199).

Lo anterior nos lleva a explorar el problema de la doble moral, y el caso de Ann Yearsley puede servir de ejemplo. Cuando se descubrió que una de sus novelas había sido escrita por a Farmer's Daughter in Gloucestershire (Spencer: 7), sus críticos la acusaron de perder el tiempo escribiendo obras de ficción en vez de dedicarlo a empresas más meritorias, como el trabajo doméstico: "When a farmer's daughter sits down to read a novel, she certainly mispends her time, because she may employ it in such a manner as to be of real service to her family: when she sits down to write one, her friends can have no hope of her" (Spencer: 34).

Un buen número de autoras del siglo XVIII, y algunas del XVII, escribieron para mantener económicamente a sus familias. Los escritores monopolizaban el mercado literario y no veían con buenos ojos que las mujeres, carentes de una educación formal, publicaran y, además, con éxito. Sin embargo, a finales del siglo XVIII las obras escritas por mujeres tuvieron tal auge que algunos autores emplean pseudónimos femeninos para poder publicar sus obras (Showalter, A Literature: 17). Afirma Elaine Showalter que, alrededor del año 1840 , esta costumbre toma otros rumbos y son las mujeres las que deben ocultarse bajo pseudónimos masculinos para poder publicar.

J. M. S. Tompkins destaca en The Popular Novel in England 1770-1800 que la mayoría de las novelas epistolares del siglo XVIII tuvieron la autoría de mujeres; asimismo, Dorothy Blakey señala en The Minerva Press 1790-1820 que esta imprenta 
publicó más novelas escritas por mujeres que por hombres (cit. en Todd: 310); Ian Watt sostiene en The Rise of the Novel (Watt 339-340) que la mayoría de las novelas del siglo XVIII fueron escritas por mujeres:

[Jane Austen's novels] reflect the process whereby, as we have seen, women were playing an increasingly important part in the literary scene. The majority of eighteenth century novels were actually written by women, but this had long remained a purely quantitative assertion of dominance; it was Jane Austen who completed the work that Fanny Burney had begun, and challenged masculine prerogative in a much more important matter. Her example suggests that the feminine sensibility was in some ways better equipped to reveal the intricacies of personal relationships and was therefore a real advantage in the realm of the novel. The reasons for the greater feminine command of the area of personal relationships would be difficult and lengthy to detail; one of the main ones is probably that suggested by John Stuart Mill's statement that 'all the education that women receive from society inculcates in them the feeling that the individuals connected with them are the only ones to whom they owe any duty'.

Por su parte, Myra Reynolds señala en The Learned Lady in England 1650-1760: "men were able to imitate, and even to usurp, female experience" (Reynolds: 89-91). Elaine Showalter, en A Literature of their Own, afirma que Oliver Goldsmith abrigaba la sospecha de que un buen número de escritores publicaron con pseudónimos femeninos novelas sentimentales, libros sobre el cuidado de los niños, partería, economía doméstica y cocina (Showalter, A Literature: 17). Dale Spender nos habla en Mothers of the Novel (Spender: 5-6) de aquellas novelistas que dieron a conocer las experiencias de la mujer, ocupando así un lugar destacado en la tradición literaria de fines de los siglos XVII y XVIII. Afirma también que resulta muy sospechoso que toda una tradición femenina de la novela desapareciera en favor de cinco autores: Daniel Defoe, Henry Fielding, Samuel Richardson, Tobias Smollett y Laurence Sterne. O en palabras de Ellen Moers: "almost uninterruptedly since the Interregnum, a small group of women have enjoyed dazzling literary prestige during their own lifetimes, only to vanish without trace from the records of posterity" (Moers: 29).

La novela constituye una extensión lógica del papel de la mujer en la sociedad de esos siglos. Una de las principales formas de expresión de la feminidad se realiza a través de cartas. Resulta, por tanto, interesante pensar que ésta fuera una de las causas principales que dieron origen a la novela epistolar. A la mujer se le niega educación y participación en el mundo público, pero se le permite escribir cartas, y éste resulta un medio apropiado para la expresión de sus ideas y sentimientos. Es entonces que la mujer halla la oportunidad de transformar una ocupación privada en una actividad pública y profesional; de participar en la creación de una nueva forma literaria y de adquirir una voz. Es por esto que no resulta sorprendente su participación activa en el origen y desarrollo de la novela.

Una razón del éxito de la novelista y la escritora del siglo XVIII es que sus obras satisfacen las necesidades de las lectoras. Muchas mujeres, excluidas de actividades 
sociales, políticas y económicas, reconocen que lo escrito por otras mujeres brinda sentido a su existencia. Estas obras les abren las puertas a un mundo de ideas y constituyen un medio para establecer relación con otras experiencias vistas desde una óptica femenina (Spencer: 17-18).

Pero la mayor limitante para estas escritoras es, sin duda, la doble moral (doublestandard ) con la que se juzga a la mujer. Lo que una mujer escribe resulta, a los ojos de la sociedad, autobiográico. Así que una dama siempre está en riesgo de quedar expuesta al ridículo o al rechazo al manifestar su intimidad al público. Una escritora, según las buenas costumbres, no debe ambicionar la fama, pues es un deseo que va en contra de la visión idealizada de la virtud femenina, específicamente, la modestia y el recato. Publicar es un acto de rebeldía que puede traer como consecuencia la hostilidad y el ridículo. Para ilustrar más claramente lo anterior, recordemos lo siguiente. A muchas mujeres que tuvieron el atrevimiento de publicar con éxito sus obras se les llamó despectivamente scribbling women, en otras palabras, escritorzuelas. El yugo de un ideal femenino ejerce una influencia determinante en la vida de la mujer y la coloca frente a una paradoja que surge de la doble moral. Las mujeres luchan con denuedo para no ser consideradas intelectuales (en otras palabras: vanidosas, atrevidas y pedantes). Son el objeto de la crítica y del ridículo por seguir conductas impropias para una dama. A este respecto señala Antonia Fraser:

For women, fame was certainly not something that was sought as it was far more closely associated with infamous than famous. To acquire a reputation these days may be for both sexes a plus in literary terms, but in the seventeenth and eighteenth centuries - and even into the nineteenth century according to Harriet Martineau in her Autobiography (1877) - for a woman to have any sort of a reputation was for her to have a bad name and to be beyond the pale. To seek public attention — which was precisely what the publication of a book entailed - was for a woman to lay herself open to every charge of indecency (Fraser: 95).

Lo anterior quizás explique la actitud un tanto incómoda que las primeras novelistas tuvieron hacia su profesión. Muchas recurrieron al estereotipo de la mujer desvalida para ganar la protección de escritores, críticos y editores. Esta actitud prevaleció hasta el siglo XIX cuando muchas mujeres prefirieron ocultar su identidad y publicar de manera anónima o con pseudónimos masculinos. Éste es el caso de Mary Brunton, autora de la novela Emelina, quien en 1810 relata sus razones para permanecer en el anonimato:

I would rather, as you well know, glide through the world unknown, than have (I will not call it enjoy) fame, however brilliant, to be pointed at,- - to be noticed and commented upon - to be suspected of literary airs - to be shunned, as literary women are, by the more unpretending of my own sex; and abhorred as literary women are, by the pretending of the other! - my dear, I would sooner exhibit as a rope-dance! (cit. en Showalter, A Literature: 17). 
Resulta difícil estudiar la tradición literaria femenina de los siglos XVII y XVIII sin referirnos a la situación política, económica y social de la mujer, en especial, como ya se mencionó, el tema de la educación, por ser decisivamente diferente de la del hombre. Un aspecto social y económico que influye de manera determinante en la desigualdad entre los géneros reside en las leyes que prohiben a la mujer poseer propiedades. Esta situación no sufre modificaciones hasta la aprobación de la "Married Women's Property Act" (1875), casi al término del periodo victoriano. Antes de dicha aprobación, la personalidad legal de la mujer quedaba al "amparo" de la common law que decretaba una unión indisoluble, tanto social como económica, de la esposa con el esposo. Como consecuencia de ello, las propiedades de la mujer se consideraban parte del patrimonio que establecía el contrato matrimonial:

[...] the professional and social emancipation of women went forward on the lines advocated by Mill's Subjection of Women (1869); women's colleges were founded at Oxford and Cambridge and women's secondary schools were much improved; the Married Women's Property Act released the wife, if she had money of her own, from economic bondages to her husband: the 'equality of sexes' began to be advocated in theory, and found its way increasingly into the practice of all classes (Trevelyan: 565).

En el Siglo de las Luces el contrato matrimonial planteaba, de acuerdo con George Duby y Michelle Perrot en Historia de las mujeres en Occidente, la contradicción del discurso ilustrado: "el matrimonio se concibe como un contrato voluntario, pero, en realidad, descansa sobre un contrato de sumisión. El siglo que rechaza que un hombre contraiga un contrato para someterse, que denuncia toda teoría que fundamenta la esclavitud en una voluntad, admite, sin embargo, la existencia de un contrato de servidumbre entre la mujer y su señor" (Duby: 92).

En el siglo XVIII se excluye a la mujer de participar en transacciones relacionadas con bienes y propiedades. Estas actividades forman parte de un mundo masculino muy alejado del ámbito de la mujer. Ruth Perry, en Women, Letters and the Novel, señala que esta situación no siempre fue así; en la Edad Media las mujeres disfrutaron de una posición económica y social mucho más ventajosa:

Although women could not serve as judges, or juries, or in government councils, a widow could obtain some degree of power, for she usually received a third part of her husband's estate, and in 1215 King John promised such widows a measure of freedom from royal authority: they were no longer forced to remarry according to the king's wishes. [...] No medieval husband could dispose of his wife's property without her free consent. Nor was a woman's inheritance treated like a dowry; each could manage her own inherited holdings as would a single woman, obligated only to feudal lords. If a husband mismanaged his wife's property or deprived her of her due proceeds from it, she had legal means to protect herself. Women had separate legal rights from her husbands' to make petitions and testify in court: man and wife were not legally considered "one". [...] The wives of middle-class English farmers were responsible for looking after their husbands' stock, making butter and cheese, helping in the fields, 
and producing cloth for the family from the wool of their own sheep. From the evidence of manorial court records, it is also clear that many widows independently farmed the holdings of their deceased husbands and engaged in litigation on their own behalf. Moreover, country women brewed and sold ale at a profit, while bourgeois and working-class women in towns and cities earned considerable sums of money from two other major home industries - textiles (spinning and weaving) and food production (brewing and baking); wives and so-called femmes soles (widows and unmarried women) also sometimes worked as leather sellers, wool merchants, shopkeepers, chandlers, shoemakers, bookbinders and goldsmiths... In the early Middle Ages, women were often active members of craft guilds, but as the era progressed, female workers were less frequently admitted as full members of these organizations (Perry: 27-32).

En el siglo XVIII la influencia del filósofo John Locke (1632-1704) tuvo una importancia decisiva. Locke expone su teoría sobre la adquisición del conocimiento en $A n$ Essay Concerning Human Understanding (1690) y sus ideas sobre educación en Some Thoughts Concerning Education (1693). En ellos propone, a grandes rasgos, que todo lo que tenemos en el pensamiento constituye un reflejo de aquello que hemos visto $\mathrm{u}$ oído, por lo tanto, nuestra conciencia es una tabula rasa en la cual se inscribe aquello que nuestros sentidos llegan a aprehender. La conciencia no es una receptora pasiva, afirma, ya que ordena y elabora todas las sensaciones. Asimismo, aboga además por la libertad de pensamiento, la tolerancia y la igualdad entre los sexos.

En The Eighteenth Century Feminist Mind, Alice Browne señala que Locke, en un comentario acerca del Génesis, 3.16, afirma que la sumisión y la subordinación de la esposa hacia el marido tiene su origen en un contrato matrimonial que puede variar de acuerdo con las circunstancias sociales e, incluso, llegar a abolirse:

In a comment on Genesis 3.16, Locke pointed out that not all men work by the sweat of their brow, and suggested that the wife's subordination was a matter of contract, which could be varied in different social circumstances. [...] Locke does not say a great deal about women, but what he says does not rule out the possibility of extending citizens' rights to women. He argues that women and men should receive similar educations (Browne: 20).

El siglo XVIII se distingue por su deseo de dilucidar el origen del pensamiento. La frase de Alexander Pope "The proper study of mankind is man" (cit. en Kermode: 1891) expresa el concepto fundamental de una época en la que la razón adquiere un significado y un valor unívoco e invariable. La razón es la misma para todos los sujetos; aun de los mudables principios religiosos, políticos y sociales es posible obtener algo sólido y perdurable: la razón. El siglo XVIII se llama a sí mismo el siglo de la razón y de la filosofía. A este respecto Ernst Cassirer señala en La filosofía de la Ilustración:

El siglo diecisiete consideró como misión propia del conocimiento filosófico la construcción de "sistemas filosóficos". [...] Esto se consigue cuando, mediante el 
método de la demostración y de la consecuencia rigurosa, se enlazan a la certeza primordial, de manera mediata, otros principios y, por la vía de este encadenamiento, se recorren todos los eslabones de lo cognoscible, sin que pueda destacarse de la totalidad ningún miembro de la cadena. El siglo dieciocho renunció a este género y a esta forma de deducción, de derivación y fundación sistemáticas. La Ilustración no recoge el ideal del pasado, sino que lo forma ella misma según el modelo que le ofrece la ciencia natural de su tiempo abstracción y la "definición" física, sino tan sólo la experiencia y la observación (Cassirer: 21-22).

Estas corrientes del pensamiento se ven reflejadas en la literatura: lo universal y lo ideal se transforman en la visión moderna por lo particular; por todo aquello que los sentidos y el individuo, como ser autónomo, aprehende.

El estado inglés tardó en intervenir y regular las cuestiones educativas. La educación se consideraba un asunto de índole privada o filantrópica (Trevelyan: 532). No fue hasta 1870 que el Parlamento aprueba la "Elementary Education Act", base del sistema inglés de educación. La educación media y superior no eran consideradas obligatorias, y sólo escuelas privadas como Eton y Harrow, a las que alumnos provenientes de familias acaudaladas asistían, impartían estos niveles de educación. No es sino hasta la segunda mitad del siglo XIX que se fundan algunas escuelas privadas para mujeres que ofrecen una educación similar a la de los colegios privados para hombres (Trevelyan: 534-536).

Para un pensador tan importante como Rousseau, en Emilio o de la educación, la desigualdad y la inferioridad de la mujer tiene sus raíces en la diferencia sexual y se extiende a su ser entero, en particular, a sus facultades intelectuales: "Una vez demostrado que ni en cuanto al carácter ni al temperamento están ni deben estar constituidos del mismo modo el hombre y la mujer, se infiere que no se les debe dar la misma educación. Siguiendo las instrucciones de la Naturaleza, deben obrar acordes, pero no deben hacer las mismas cosas" (Rousseau: 278).

Aquí, en realidad, no estamos en presencia de un discurso femenino sino de un discurso constituido por un sujeto distinto del femenino que se pone en su lugar. Somos testigos de una palabra doblemente masculina, ya que toma la palabra del otro sexo y la hace suya. Resulta entonces evidente que el discurso dominante que representa a la mujer se origina en una construcción masculina de la naturaleza femenina. El argumento de Rousseau es claro: la mujer se diferencia del hombre por la constitución de su cuerpo. Pero esta idea suscita una pregunta esencial a la que muchas escritoras del siglo XVIII trataron de dar respuesta: ¿la condición intelectual, moral, social, política de la mujer se basa en esta diferencia biológica o guarda relación con la educación que recibe?

Estas palabras traen en consecuencia un planteamiento de gran importancia en los debates del siglo XVIII: ¿posee la mujer la capacidad de razonar? Rousseau considera a la mujer un ser subordinado a la pasión, a las emociones, incapaz de conceptualizar. La mujer, afirma, no está desprovista totalmente de razón, pero esta facultad es en ella 
más simple que en el hombre y sólo debe cultivarse en relación con sus deberes naturales (obedecer al marido, serle fiel, cuidar el hogar y los hijos). En otras palabras, la mujer debe permanecer en el mundo cerrado de la domesticidad por ser éste el legado de la naturaleza. La única ciencia que debe conocer tiene sus bases en el sentimiento, y el objeto de sus sentimientos y emociones debe centrarse en los hombres que la rodean, en su esposo principalmente.

Las inconveniencias de ser mujer se traducen también en una servidumbre fisiológica que la persigue implacablemente hasta que finaliza su etapa de fecundidad: "No hay paridad alguna entre ambos sexos en cuanto a lo que es consecuencia del sexo. El varón sólo en ciertos instantes lo es, la hembra es toda su vida hembra, o al menos toda su juventud: todo la llama a su sexo" (Rousseau: 281).

El sexo es, ante todo, lo que legitima por naturaleza la inferioridad femenina, pues en la mujer lo que prevalece es el sexo. En el acto sexual, de acuerdo con Rousseau, el hombre es activo y fuerte, mientras que la mujer es pasiva y débil. El hombre debe poder y querer, mientras que la mujer se contenta con resistir un poco. Para el hombre, agrega, la necesidad sexual no es una necesidad física, por lo tanto, el sexo no define la naturaleza del hombre pero sí, de manera paradójica, la naturaleza femenina:

La tercera consecuencia de la constitución de los sexos es que el más fuerte sea en la apariencia el árbitro, y en la realidad dependa del más débil; y no así por un frívolo estilo de galanteo, sino por una invariable ley de la Naturaleza, que dando más frivolidad a la mujer para que excite deseos que al hombre para que los satisfaga, hace a éste dependiente, mal de su agrado, de la buena voluntad de aquélla (Rousseau: 280).

En el discurso de la Ilustración el hombre utiliza la facultad que lo define: el entendimiento y la razón. Si el discurso ilustrado se dirige a todos los seres humanos, éste adquiere una dimensión universal. Sin embargo, el discurso ilustrado es un discurso de hombres para hombres. Se considera que vale para todos pero, en realidad, es privilegio de unos pocos. En consecuencia, es excluyente; en él la mujer es sólo un objeto de estudio. El discurso ilustrado se cifra en un discurso dual: el del hombre sobre el hombre, y el del hombre sobre la mujer. A la mujer, entonces, sólo se le concede el espacio de objeto del discurso masculino. La palabra y la escritura se cargan de elementos ideológicos que derivan de la necesidad de dominio que tiene una parte del género humano sobre la otra. Al legitimizarse, este discurso dicta el destino de la mujer como un destino donde su palabra carece de valor.

El argumento que recorre varios textos del siglo XVIII con el fin de justificar la desigualdad de los sexos se apoya en la idea, pocas veces cuestionada, de que una de las partes es superior a la otra. La fuerza y la razón definen la naturaleza del hombre mientras que a la mujer la determinan los sentimientos y el deseo de atraer. Un arma consustancial al discurso de poder es la educación. Por lo mismo, es necesario tomar en cuenta las diferencias en la educación que se imparte a hombres y mujeres en el siglo de la Ilustración. En este sentido, es notable que pese a estarles vedada la palabra se 
producen un gran número de tratados sobre educación escritos por mujeres. En ellos se insiste en la necesidad de brindar un carácter práctico a la educación que se ofrece a las niñas. Pero, como una golondrina no hace verano, estos cuestionamientos que en ocasiones se convierten en denuncias, no trascienden. La creencia en la desigualdad natural de hombres y mujeres hace que prevalezcan los prejuicios vinculados a lo que debe ser la educación de la mujer y, por lo mismo, estos cuestionamientos no repercuten en el grueso de la población. El ciudadano común del siglo XVIII no vislumbra aún la influencia crucial de la educación en la vida de cada individuo y pocas son las voces que reconocen su importancia.

A pesar de todo, muchas escritoras del siglo XVIII señalan que la desigualdad entre géneros no se debe a condiciones físicas, climáticas, etcétera, sino que estriba en factores sociales y políticos que a lo largo de la historia marcaron al género humano. Estos factores traen consigo las diferencias entre hombres y mujeres, y establecen ciertas características que se atribuyen más a un sexo que a otro. La desigualdad entre los géneros se basa, en gran medida, en diferencias naturales; es el resultado de una educación deficiente que Mary Astell (1666-1731) denuncia en A Serious Proposal to the Ladies (1694):

For since God has given women as well as men intelligent souls, why should they be forbidden to improve them? Since he has not denied us the faculty of thinking, why should we not (at least in gratitude to him) employ our thoughts on himself their noblest object, and not unworthily bestow them in trifles and gaities and secular affairs? Being the soul was created for the contemplation of truth as well as for the fruition of good, is it not as cruel and unjust to exclude women from the knowledge of the one as from the enjoyment of the other? [...] A rational mind will be employed, it will never be satisfied in doing nothing, and if you neglect to furnish it with good materials, 'tis like to take up with such as come to hand (cit. en Gilbert: 116-117).

No sorprende, entonces, que un buen número de mujeres desearan tener una educación y que lo lograran por ellas mismas o mediante la orientación de algún miembro de la familia o un tutor. En el siglo XVII la educación era privilegio de unos cuantos y no es sino hasta el XVIII que surgen nuevas ideas. Sin embargo, las oportunidades para la mujer eran escasas y se privilegiaba la educación de los varones, aunque muchas hijas de familias acaudaladas compartín la educación que se brindaba a sus hermanos. Muchas mujeres aprendieron lenguas extranjeras a través de diccionarios y gramáticas, como Mary Wollstonecraft, quien para ganarse la vida tradujo del francés, holandés y alemán. En consecuencia, al percibir sus limitaciones, un buen número de escritoras manifiestan su descontento e intentan quebrar los límites que impiden el desarrollo de la educación de las mujeres. Por lo mismo, y no sin un dejo de ironía, muchas escritoras piden en sus publicaciones disculpas en los prólogos o dedicatorias y hacen alusión a sus carencias en el terreno de la educación formal. Es sorprendente descubrir cómo no se arredran ante a las prohibiciones impuestas. Piensan, dicen y escriben e, incluso, algunas publican con éxito sus manuscritos. Esto puede atribuirse a dos 
factores decisivos: el aumento en el número de lectoras y lectores, y el interés por la educación.

En el siglo XVII la mujer incursionó en el mercado literario, pero también pagó el precio de su osadía. Las damas de clase alta establecieron, de manera paralela, una tradición literaria, pero su matiz principal reside en su carácter privado. Éste es el caso de Margaret Cavendish, duquesa de Newcastle (1623-1674), Katherine Philips (16211664), Lady Mary Chudleigh (1656-1710) y Anne Killigrew (1660-1685). Según la crítica, resulta difícil precisar el número de obras escritas por mujeres, ya que muchas escribieron en manuscritos que circularon sólo entre amigas y amigos, y, como es natural, sólo el texto impreso tiene mayores posibilidades de perdurar.

En el siglo XVIII la mujer tenía mayor libertad de escribir y publicar, y esto se debe a varios factores. En este siglo la literatura se vuelve un bien de consumo y esto da pie al inicio del proceso de comercialización. Es el momento en que el editor sustituye al mecenas y al mundo de la corte; se presta especial importancia al gusto de lectores y lectoras. Por vez primera en la historia de la literatura muchas escritoras no son religiosas ni pertenecen a la clase alta o a la aristocracia, son mujeres comunes, la mayoría de clase media, que se dedican a la literatura como profesión. Resulta significativo que Virginia Woolf en Orlando (1928) nos hable de un personaje que nace en el Renacimiento y se transforma en mujer en la segunda mitad del siglo XVII, época crucial para la tradición literaria femenina y para la historia de la literatura inglesa que, como Woolf señala, coincide con el momento en que la mujer ingresa en el mercado literario:

Thus, towards the end of the eighteenth century a change came about which, if I were rewriting history, I should describe more fully and think of greater importance than the Crusades or the Wars of the Roses. The middle-class woman began to write. For if Pride and Prejudice matters, and Middlemarch and Villette and Wuthering Heights matter, then it matters far more than I can prove in an hour's discourse that women generally, and not merely the lonely aristocrat shut up in her country house among her folios and her flatterers, took to writing (Woolf: 55).

Durante el siglo XVIII se operan cambios en la sociedad inglesa que dan origen al capitalismo. Esta transformación parte de una nueva economía basada en el comercio y no en la agricultura. Por tanto, la participación económica de la mujer en la nueva sociedad capitalista toma otros rumbos. El interés por la educación y el surgimiento de profesiones para una clase media contribuyen a que la mujer se integre al nuevo mercado literario y periodístico dando inicio, como se menciona anteriormente, a una tradición literaria femenina. Los periódicos que se publican en Londres constituyen un factor muy importante de este mercado. Tal es el caso de The Daily Courant (1702) y de publicaciones periódicas (periodical essays) como The Tatler, The Spectator y The Gentleman's Magazine. En ellos se difunden artículos y cuentos, y en la ensayística predominan temas de educación, moral o conducta escritos en su mayoría por hombres que generan, con un claro propósito didáctico, una serie de opiniones sobre la mujer. 
Entre estas publicaciones periódicas encontramos un fenómeno interesante: The Female Spectator (1722-1746) de Eliza Haywood (ca. 1693-1756). Esta autora crea el primer periódico escrito por - y para - mujeres con artículos sobre educación, literatura, arte y filosofía. Uno de los temas centrales de esta publicación es la mujer y su educación en la nueva y fluctuante sociedad del siglo XVIII. En 1746 Haywood publica una carta (bajo el pseudónimo de Cleora) en la que se señala que la educación que se brinda a la mujer tiene el propósito de confinarla al ámbito de lo privado y lo doméstico. Haywood, de esta manera, subvierte el debatido tema de la educación. En su argumento central advierte que la desigualdad de la mujer es sólo una invención que tiene su origen en la educación, basada en la obediencia, la subordinación y el sometimiento:

There is, undoubtedly, no Sexes in Souls, and we are as able to receive and practise the Impressions, not only of Virtue and Religion, but also of those Sciences, which the Men engross to themselves as they can be: - Surely our Bodies were not form'd by the greater Creator out of the finest Mould, that our Souls might be neglected like the coarsest of the Clay.

[...] The Objection, therefore, that I have heard made by some Men, that Learning would make us too assuming, is weak and unjust in itself, because there is nothing would so much cure for those Vanities we are accused of, as Knowledge.

[...] O but, say they, Learning puts the Sexes too much on an Equality, it would destroy that implicit Obedience which it is necessary the Women should pay to our Commands: - If once they have the Capacity of arguing with us, where would be our Authority!

Now I will appeal to any impartial Reader, even among the Men, if this very Reason for keeping us in Subjection does not betray an Arrogance and Pride in themselves, yet less excusable than that which they seem so fearful of our assuming (Mahl: 235-236).

La dinámica y multifacética sociedad inglesa del siglo XVIII posee una visión moderna por lo particular y por el individuo, que se expresa a través del realismo, el cual brinda una nueva libertad discursiva. A partir de la segunda mitad del siglo XVIII se construyen en torno a la mujer imágenes de benevolencia, generosidad y receptividad emocional que aluden de manera directa a las virtudes de la clase media, entre las que destacan la diligencia, la perseverancia y la integridad moral. Lo anterior resulta evidente en Pamela (1740) de Samuel Richardson, en donde la protagonista sirve de modelo a la nueva imagen de la mujer y de las virtudes de la clase media.

La novela trajo consigo cambios que repercuten de manera decisiva en las escritoras. El sistema de patronazgo que subordinaba a los escritores al gusto de un número reducido de lectores desaparece y surge, en su lugar, un amplio público de lectores y lectoras que muestran interés por una gran variedad de temas. Esta nueva diversidad beneficia a un buen número de escritoras que irrumpen en el mercado literario. A partir de la Restauración varias dramaturgas como Aphra Behn (1640-1689) y Susannah Centlivre (ca.1667-1723) publican sus obras. En 1688 Aphra Behn publica Oronooko, 
considerada por varias críticas contemporáneas la primera novela inglesa; es, además, la obra precursora de la ficción escrita por mujeres. Mary Delarivière Manley (ca. 16671724) y Eliza Haywood (ca. 1693-1756) siguen su ejemplo. Estas primeras novelistas descubren que la literatura puede ser una de las pocas profesiones lucrativas para la mujer; sin embargo, tanto sus publicaciones como sus vidas padecieron el juicio de la doble moral.

A finales del siglo XVII la mujer dependía del consentimiento de esposos, amigos o parientes para publicar sus obras. Tal es el caso de poetas como Katherine Philips (1631-1664), quien firmaba con el pseudónimo The Matchless Orinda, casada con un acaudalado comerciante puritano, cuya obra causó admiración a Abraham Cowley y John Dryden. En las colonias americanas surge la primera poeta norteamericana de raza negra Phillis Wheatley (ca. 1753-1786). Su poesía, de tema religioso, se publicó primero en Londres con la ayuda de la condesa de Huntington en un volumen titulado Poems on Various Subjects, Religious and Moral (1773). Anne Bradstreet (ca. 16121672), otra poeta norteamericana, hija de un puritano acaudalado, Thomas Dudley, emigró en 1630 a las colonias norteamericanas con su familia. En 1650 su cuñado, John Woodbridge, publicó en Londres una colección de poemas bajo el título The Tenth Muse, y en el prólogo aclara que sus poemas eran el resultado de "the work of a woman, honoured and esteemed where she lives, for her exact diligence in her place, and discreet managing of her family occasions, and these poems are but the fruit of some few hours, curtailed from her sleep and other refreshments" (cit. en Gilbert: 60).

En "The Prologue", poema que inicia esta colección, Anne Bradstreet habla con humildad de su pobre talento, al que considera inferior al del hombre. Pero en sus palabras encontramos la velada ironía que a ratos se revela a través de un relámpago de ira:

1

To sing of wars, of captains, and of kings,

Of cities founded, commonwealths begun,

For my mean pen are too superior things:

Or how they all or each their dates have run,

Let poets and historians set these forth;

My obscure lines shall not so dim their worth.

5

I am obnoxious to each carping tongue

Who says my hand a needle better fits;

A poet's pen all scorn I should thus wrong,

For such despite they cast on female wits;

If what I do prove well, it won't advance,

They'll say it's stolen, or else it was by chance.

(cit. en Gilbert: 61-62).

Estas autoras que hemos mencionado constituyen unos eslabones más de la cadena que nos llevará al siglo XVIII, donde la escritora goza de una mayor libertad. Una en- 
sayista como Elizabeth Robinson Montagu (1720-1800), figura prominente del grupo de las bluestockings (la historiadora Doris Stenton afirma que este término tiene su origen en el tono informal de las reuniones organizadas por damas adineradas interesadas en la literatura, y a las que los caballeros podían asistir "even in their blue stockings") (cit. en Gilbert: 56), negocia personalmente sus publicaciones con los editores. La ensayista Hannah More (1745-1833) y la novelista Charlotte Smith (1749-1806) disfrutan de una gran popularidad, y con sus publicaciones mantienen, en gran medida, a sus familias. La mayoría de sus lectores son mujeres de clase media interesadas en temas relacionados con la esfera de lo doméstico y lo privado, mujeres que tienen suficiente dinero y tiempo libre para hacer uso de las bibliotecas circulantes. En el siglo XVIII la mujer ingresa al mercado literario en el momento en que ser escritor(a) se convierte en una profesión remunerada; sin embargo, muchas de las obras escritas por mujeres no forman parte de la tradición literaria dominante pues la crítica las excluye calificando su producción de "intentos aislados". La doble moral con que se juzga a la mujer puede ser una de las causas, y esta actitud agudiza aún más las diferencias entre el ámbito de lo masculino y lo femenino.

Es así que los argumentos sobre la igualdad moral e intelectual de mujeres y hombres resultan difíciles de conciliar con la doble moral. La doble moral juzga a la mujer como "mala" o "buena" a partir de un comportamiento sexual aceptado por la sociedad, sin consideraciones de otra índole. Recordemos, en voz de Virginia Woolf, los prejuicios en contra de Aphra Behn, pieza esencial en la historia de la escritura femenina:

\begin{abstract}
With Mrs Behn we turn a very important corner on the road. [...] We come to town and rub shoulders with ordinary people in the streets. Mrs Behn was a middle-class woman with all the plebeian virtues of humour, vitality, and courage; a woman forced by the death of her husband and some unfortunate adventures of her own to make her living by her wits. She had to work on equal terms with men. The importance of that fact outweighs anything that she actually wrote, even the splendid A Thousand Martyrs I have made, or Love in Fantastic Triumph sat, for here begins the freedom of the mind, or rather the possibility that in the course of time the mind will be free to write what it likes. For now that Aphra Behn had done it, girls could go to their parents and say, You need not give me an allowance; I can make money by my pen (Woolf: 54).
\end{abstract}

La mayoría de las escritoras del siglo XVIII no pusieron en duda la doble moral, y en su afán por ser consideradas mujeres "buenas" aceptaron la castidad como una condición necesaria de la mujer virtuosa. Si se calificaba a una escritora de ser una "mujer mala", los obstáculos para una de las pocas profesiones aceptadas para la mujer de clase media resultaban insalvables. Los severos juicios en contra de la mujer que infringiera la moral establecida trajeron, en consecuencia, la aceptación tácita de los prejuicios de la doble moral.

El argumento central de la doble moral proclamaba la castidad como una virtud esencial en la mujer. Las reglas y restricciones morales y sociales regulaban la conducta de la mujer, alentaban su pasividad y la circunscribían a la esfera de lo privado, 
protegiendo así su virtud de los desenfrenos de su naturaleza (recordemos el Emilio de Rousseau). El tema de la castidad que escritoras como Mary Astell y Catherine Macaulay Graham abordaron nos muestra el poder de la doble moral. La doble moral influye poderosamente en el discurso mismo de la mujer, creando también en él una doble moral. El discurso femenino se torna así tan excluyente como el del hombre; se dirije tan sólo a la mujer virtuosa y racional, es decir, a la imagen creada de la figura femenina convencional:

A woman must be wise and good and much above what we suppose the Sex capable of, I fear much greater than e'er a Man can pretend to, who can so constantly conquer her Passions, and divest her self even of Innocent Self-love, as to give up the Cause when she is in the right, and to submit her enlightened Reason, to imperious Dictates of a blind Will, and wild Imagination, even when she clearly perceives the ill Consequences of it, the Imprudence, nay Folly and Madness of such a Conduct (cit. en Perry: 59).

La doble moral se impone a los deseos naturales de hombres y mujeres; es el resultado de restricciones y leyes externas, y aunque algunas voces se elevan en contra de su injusticia — como es el caso de Mary Wollstonecraft—, éstas no son escuchadas. La mujer que cometía "pecados" sexuales era más culpable que el hombre, y las sanciones, por consiguiente, más severas. Así, la virtud de la mujer se transforma en el paradigma moral de la sociedad del siglo XVIII, en especial durante la segunda mitad de este siglo, y las consecuencias sociales y económicas de sus actos se someten a un cuidadoso control.

\section{Obras citadas}

Browne, Alice. The Eighteenth Century Feminist Mind. Londres: The Harvester Press, 1987. Impreso.

CASSIRER, Ernst. La filosofía de la Ilustración. México: FCE, 1984. Impreso.

Duby, George y Michelle PERrot. Historia de las mujeres en Occidente. Vol. 6. Trad. Marco Aurelio GalmarinI. Madrid: Taurus Ediciones, 1993. Impreso.

Fraser, Antonia. The Weaker Vessel: Woman's Lot in the Seventeenth Century England. Nueva York: Vintage Books, 1994. Impreso.

GILBERT, Sandra M. y Susan GuBAR, eds. The Norton Anthology of Literature by Women. Nueva York: W.W. Norton \& Co., 1985. Impreso.

Kermode, Frank, ed. The Oxford Anthology of English Literature. Vol. I. Nueva York: Oxford University Press, 1973. Impreso.

Lennox, Charlotte. The Female Quixote or the Adventures of Arabella. Nueva York: Oxford University Press, 1989. Impreso.

Mahl, Mary R. Helene Koon. The Female Spectator: English Women Writers before 1800. Bloomington: Indiana University Press, 1977. Impreso. 
Moers, Ellen. Literary Women. Nueva York, Doubleday, 1985. Impreso.

Perry, Ruth. The Celebrated Mary Astell. Chicago: The University of Chicago Press, 1986. Impreso.

REYNOLDS, Myra. The Learned Lady in England 1650-1760. Gloucester: Peter Smith, 1964. Impreso.

Rousseau, Juan Jacobo. Emilio o de la educación. México: Porrúa, 1993. Impreso.

Showalter, Elaine. "Toward a Feminist Poetics". The New Feminist Criticism. Essays on Women, Literature and Theory. Ed. Elaine SHOWALTER. Nueva York: Pantheon Books, 1985. Impreso.

. A Literature of their Own. Princeton: Princeton University Press, 1977. Impreso.

SPENCER, Jane. The Rise of the Woman Novelist: From Aphra Behn to Jane Austen. Oxford: Basil Blackwell, 1987. Impreso.

SPENDER, Dale. Mothers of the Novel. Nueva York: Pandora, 1986. Impreso.

ToDD, Janet, ed. Gender and Literary Voice. Nueva York, Holmes \& Meier, 1983. Impreso.

Trevelyan, G. M. English Social History. Harmondsworth: Penguin Books, 1970. Impreso.

WatT, Ian. The Rise of the Novel. Berkeley, University of California Press, 1984. Impreso.

Woolf, Virginia. A Room of One's Own and Three Guineas. Londres: Viking, 2001. Impreso. 
